\title{
Disease appellation: insistence on the necessity of thoughtfulness for deep impacts
}

\section{Letter to the Editor}

Several research groups worldwide with expertise in different fields of medical sciences have been working continuously in order to discover new treatments for the mounting number of challenging diseases with considerably increasing prevalence, such as Alzheimer' $\mathrm{s}$ disease, different types of cancer, diabetes, heart diseases, etc. Obviously, to these illnesses and most of other health problems, a corresponding name is attributed and globally used by scientists all over the world. The World Health Organization (WHO) with other organizations such as World Organization for Animal Health and the Food and Agriculture Organization of the United Nations have been monitoring and controlling this issue, especially for the new emerging diseases. ${ }^{1}$

The appellation of a disease has become regarded more significantly in the recent few years. I am writing this paper to draw more attention to the importance of the appropriate selection of a disease name, particularly for its impact on the patient and on countries' social, cultural and economic state. Although only scientific researchers, pharmacists, doctors and other experts in the field of medicine are directly involved in science promotion and have command of the scientific and technical language, the names of diseases are used by the whole population, and in some cases created by people who are out of the field for common use. In addition almost everyone is concerned with diseases, as a scientist, a patient or a caregiver because of the mounting prevalence of a tremendous number of illnesses. ${ }^{2}$ Whilst experts are aware of the disease underlying mechanisms, and they utilize its appellation as a practical tool for international scientific communication and knowledge exchange and promotion, the other part of the society may not always consider the names of a disease under the same measures. The names of the diseases are not only mentioned in published scientific papers and academic meetings and conferences, and not only utilized by experts in medical sciences. The whole population might have a common name for each disease. The problem is that the public may use inappropriate names for certain diseases, and most of them, for instance, cannot even dare to say the word "cancer" because they may believe that it can affect the person through pronouncing its name, and in several regions in the world, they may attribute to this disease other names such as "that disease", "the bad disease" or "the disease that has no treatment" and others may classify it into "male cancer" and "female cancer" according to cancer's advancement state. People, particularly in some regions where the level of education is relatively low, may further give certain names to some diseases describing their process as they imagine it. For example, some people believe in the existence of an organ like a leaf in the brain and saying that it loses its balance in the brain of the person suffering from a mental disease, and they transfer this wrong information to next generations that will be completely misguided, unfortunately.

All these inappropriate appellations and ideas about diseases might have certainly a deep impact on many sides. Most importantly, the patients, who are already suffering from a disease, might have
Volume 2 Issue 5 - 2015

\author{
Besma Boubertakh 1,2 \\ 'Key Laboratory of Animal Models and Human Disease \\ Mechanisms of Chinese Academy of Sciences \& Yunnan Province, \\ Kunming Institute of Zoology, Kunming, Yunnan 650223, PR \\ China \\ ${ }^{2}$ University of Chinese Academy of Sciences, Beijing 10049, PR \\ China
}

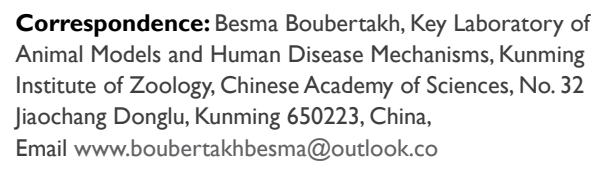

Correspondence: Besma Boubertakh, Key Laboratory of Animal Models and Human Disease Mechanisms, Kunming Institute of Zoology, Chinese Academy of Sciences, No. 32 Jiaochang Donglu, Kunming 650223, China, Email www.boubertakhbesma@outlook.co

Received: October 27, 2015 | Published: November 18, 2015

another burden caused by an inconsiderate appellation of their illness; for instance one who is suffering from cancer and fighting against its great challenges, would certainly suffer more with others naming their disease as the one without treatment or other thoughtless appellations. These inconsiderate appellations might have further impact on communication between persons when they describe mistakenly to describing the disease mode of transmission for instance, and may influence even the economic state of a country that had in a certain period an epidemic disease for example which led to attributing to it the name of a city for instance, which may influence deeply tourism in the region concerned, and could also bear a wrong information misguiding that that disease is only limited to a certain region or a certain country in the globe. Naming a disease after the scientist who discovered it, might bear a noble meaning of gratitude and recognition for their achievements, however, I suggest that this might have on the others side certain negative influence for instance for the family of the scientist and its next generations, who bear the same name and would suffer from being called by the name of a disease.

The patient has an urgent need for encouragement and support in order to overcome the difficulties of their condition, hence the importance of comprehensive sensitization of the whole population about using only scientific names determined by qualified experts for all the diseases. This would help significantly to avoid the negative impacts of arbitrarily-attributed disease names and misleading concepts about their properties. Recently, the WHO with other organizations has done great efforts to solve these problems influencing the whole society. The appellation of a disease could have further and deeper negative impacts since it may have also an undoubted influence on tourism, whole economy and cultural exchange between different countries when a disease bears the name of a city or a region for instance. For these reasons, the scientific community, different associations, and the media, especially social media, which has gained great power of influencing thoughts of persons of different ages, should cooperate efficiently towards providing a better environment for the patient via encouraging and sensitizing about the importance and the necessity 
of utilizing only scientific names attributed by experts in the field. Furthermore, scientists with expertise and qualifications for disease appellation are invited to take into consideration all the possible impacts of the selected names for diseases, socially, culturally and economically.

\section{Acknowledgements}

None.

\section{Conflict of interest}

The author declares that there are no conflicts of interest.

\section{References}

1. WHO issues best practices for naming new human infectious diseases; 2015.

2. Boubertakh B. More Care for patient's caregiver training: disregarded necessity besides pharmaceutical research. J Psychol Clin Psychiatry. 2015;4(1):00182. 